\title{
La critica di Benedetto XVI al relativismo odierno
}

\author{
Peter Ivanecký \\ PONTIFICIA UNIVERSITÀ DI SAN TOMMASO D'AQUINO, ROMA \\ UNIVERSIDAD CATÓLICA DEL MAULE, TALCA \\ pet.ivanecky@gmail.com
}

A partire dall'anno 2005 fino alla fine dell'anno 2011, cioè in circa sette anni, la frequenza con cui appare termine 'relativismo' negli scritti del Magistero è paragonabile alla frequenza della ricorrenza dello stesso termine nei 26 anni e 5 mesi del pontificato di Giovanni Paolo II. Nell'anno 2005 Joseph Ratzinger diventerà il vescovo di Roma. Associamo quindi alla figura di questo pontefice la ripetizione così frequente del termine relativismo e in questo scritto cercheremo di capire le ragioni di tale forte accento sul relativismo nel suo Magistero pontificio. Porremo dunque al centro della nostra analisi esclusivamente il termine 'relativismo' e le sue accezioni, e non ci preoccuperemo di prendere in considerazione gli altri paradigmi storici che troveremo accanto ad esso. Sottolineiamo così il fine di questo lavoro, che consiste nella comprensione della critica di Benedetto XVI al relativismo, così come appare dai suoi discorsi. Il testo così concepito -il primo del suo genere negli studi su Joseph Ratzingerpotrebbe essere caratterizzato, per sua natura, come una introduzione al tema e potrebbe con ciò fornire alcuni dati a altri studi, sia filosofici che teologici, sul pensiero di papa Benedetto XVI. Anticipando possiamo svelare che il relativismo apparirà dopo la morte dell'esperimento marxista, e che la critica chiave del teologo e papa Ratzinger riguarderà uno dei cardini del sistema democratico, la maggioranza.

L'esposizione sul relativismo nel pensiero di Benedetto XVI sarà fatta mediante la presentazione dei testi nei quali egli accenna implicitamente al tema o lo tratta esplicitamente. Con un percorso cronologico di alcune opere di Joseph Ratzinger vogliamo indicare a tre periodi differenti in cui si presentano altrettante evoluzioni del tema da parte sua. L'impiego di questo metodo mostrerà la precisa apparizione di certi discorsi in cui 
egli sviluppa, matura e definisce il concetto del relativismo. In questo modo analizzeremo il periodo che va dalla metà degli anni settanta fino agli anni ottanta; successivamente il periodo degli anni novanta che terminerà con la sua elezione pontificia, e infine il periodo del pontificato. Quando invece si tratterà dei testi del Magistero, la nostra ricerca consisterà nel rilevare la presenza quantitativa del termine 'relativismo', di cui porteremo alla luce le sue svariate accezioni.

1. Analisi dell'occorrenza del termine 'Relativismo' nel magisteRo DOtTRinale di Giovanni PaOlo II.

Motiviamo l'inclusione dell'analisi sul relativismo nel magistero di Giovanni Paolo II con le seguenti riflessioni. È comunemente risaputo che Joseph Ratzinger ha strettamente collaborato con il magistero dottrinale di Giovanni Paolo II $^{1}$. Perciò, può darsi, che il concetto del relativismo non è proprio di Benedetto XVI, ma egli lo prende in prestito da un concetto già presente nella dottrina magisteriale. Sono da considerare anche altre possibilità, e cioè che Giovanni Paolo II affidi alcune trattazioni sul relativismo a colui che è già il suo prefetto preferito, o che insieme approfondiscano un certo discorso sul relativismo. Per confermare una di queste supposizioni, crediamo sia necessario fare una concisa ricerca dell'apparizione del termine relativismo nei testi del Magistero precedente. Ciò sarà fatto individuando l'occorrenza del termine in tre differenti tipi di pubblicazioni che contengono testi del Magistero, per poi racchiuderlo in diverse categorie a seconda delle sue accezioni. $\mathrm{Da}$ questa indagine rileveremo alcune osservazioni che serviranno per la successiva analisi sul relativismo nel pensiero di Joseph Ratzinger. La prima ricerca dell'occorrenza del termine 'relativismo' è stata fatta sugli Acta Apostolicae Sedis, da cui riportiamo le seguenti accezioni che sono circa cento.

1) Il relativismo e le questioni delle religioni. Il termine viene qui sviluppato in rapporto al pluralismo religioso, al rischio del sincretismo o

\footnotetext{
Alla domanda sulla collaborazione con Giovanni Paolo II il cardinale Ratzinger risponde: «In questo senso ho collaborato anch'io al magistero dottrinale del papa e vi ho in qualche modo contribuito, cosa che, sicuramente, ha caratterizzato questo pontificato. Ma il papa ha comunque la sua linea», in J. RATZINGER: Il sale della terra. Cristianesimo e la Chiesa cattolica nella svolta del millenio (San Paolo, Cinisello Balsamo 1997) 122-123.
} 
al pericolo delle sette e dei nuovi culti emergenti. Quando nei testi si parla di tali temi, contestualmente è menzionata frequentemente anche la mentalità indifferentista. Qui emergono due concezioni che vengono ripetute in vari documenti: la prima afferma che una religione vale l'altra, mentre con la seconda si sostiene che nel dialogo tra le religioni il relativismo e l'indifferentismo devono essere evitati ${ }^{2}$.

2) Il relativismo morale. Tale concetto apparirà e si chiarirà nei documenti gradualmente. Durante gli anni ' 80 il termine è usato soltanto tre volte nel pontificato di Giovanni Paolo II. Ma il concetto è presente quando si parla in generale del relativismo, riferito alla caduta del senso del peccato, alla relativizzazione della norma morale, al rovesciamento etico ed a simili temi etici ai quali più tardi si applicherà l'aggettivo 'morale's. Si constata inoltre che il relativismo morale è il risultato dello storicismo ${ }^{4}$. A partire dagli anni '90 il termine relativismo morale è frequentemente presente quando si elencano fenomeni della società attuale; annotiamo che il termine raramente viene riportato come isolato; nella stragrande maggioranza dei riferimenti esso è riportato accanto al relativismo dogmatico, religioso e culturale, alla chiusura egoistica, accanto al secolarismo e all'indifferenza religiosa ${ }^{5}$. Negli anni '90 si associa al relativismo un tema nuovo, e cioè quello concernente la democrazia. Il relativismo morale è un pericolo per la democrazia e le sue condizioni sono incompatibili con essa ${ }^{6}$. In altre apparizioni del termine, che vanno dall'anno 2000 fino alla fine del pontificato, si approfondisce la coscienza di una crisi della civilizza-

2 Cf. Acta Apostolicae Sedis. Commentarium officiale, (Typis Polyglottis Vaticanis, 1981-2007), 72-99 [1980-2007], qui 73 [1981], 295 (da qui in poi userò l'abbreviazione AAS), AAS 77 [1985], 1147, AAS 81 [1989], 94, AAS 83 [1991], 281, AAS 86 [1994], 493, 498, AAS 88 [1996], 555, AAS 92 [2000], 358, 763, AAS 93 [2001], 382, AAS 95 [2003], 332, 683, AAS 96 [2004], 22, 475, 752, 787, 796,917 . In vista degli obiettivi della presente ricerca non riteniamo opportuno soffermarsi sulle caratteristiche specifiche e contestuali dei discorsi o discernere tra vari tipi dei documenti del Magistero, perciò nella citazione dei documenti magisteriali non saranno riportate le informazioni relative a ciò.

3 Cf. AAS 77 [1985], 226, 591, AAS 78, [1986], 348, 1100, AAS 79 [1987], 1474, AAS 80 [1988], 765, 1311.

4 Cf. AAS 77 [1985], 226, AAS 78, 1986, 348.

5 Cf. AAS 83 [1991], 984, AAS 85 [1993], 499, 692, AAS 86 [1994], 493, 498, AAS 88 [1996], 492, AAS 90 [1998], 811.

6 Cf. AAS 89 [1997], 167, AAS 91 [1999], 204-205; cf. AAS 83 [1991], 850, AAS 85 [1993], 909, AAS 87 [1995], 481-482. 
zione, se ne individuano le cause nel relativismo morale e sui suoi effetti sulla società e sulla chiesa ${ }^{7}$.

3) Il termine relativismo senza alcuna specificazione. Esso presenta la maggiore occorrenza, tra tutte quelle del termine, e cioè ventinove volte. Al secondo posto annotiamo il relativismo morale, che appare diciannove volte in tutti gli AAS. Il termine relativismo è dapprima usato nei testi del Magistero quando si elencano i pericoli della società contemporanea; esso è quindi riportato accanto ad altri pericoli quali il materialismo, il pragmatismo, il consumismo, l'edonismo, e così via, e nella maggior parte dei casi esso non viene chiarificato ulteriormente ${ }^{8}$. Successivamente, alla fine degli anni '90, il relativismo non è più un pericolo per la società, ma è proprio della cultura contemporanea. Occorre dire che l'apparenza del termine varia poco e soltanto in relazione all'uditorio; il termine in sostanza presenta lo stesso concetto: il relativismo è un pericolo, un male, un rischio per la società.

4) Annotiamo otto volte la presenza dell'espressione relativismo etico, e ciò assai tardi, soltanto a partire dall'anno 1993. Tale espressione cinque volte esprime il fenomeno del tempo presente o la minaccia di questo ${ }^{9}$. Tre volte, invece, esso fa parte del discorso sulla democrazia ${ }^{10}$.

5) Altre caratteristiche di relativismo. Due volte viene menzionato il 'relativismo scettico', 11 due volte troviamo l'espressione 'relativismo di comodo', ${ }^{12}$ due volte si parla del 'relativismo filosofico', ${ }^{13}$ mentre una volta del 'relativismo della verità e della morale' riguardo alla vita del-

Cf. AAS 93 [2001], 517, AAS 94 [2002], 31, AAS 95 [2003], 258, 599-600, 694, AAS 96 [2004], 479, 484.

8 Cf. AAS 74 [1982], 1258, AAS 79 [1987], 1461, AAS 80 [1988], 819, 1153, 1461, AAS 82 [1990], 1564, AAS 83 [1991], 408, AAS 84 [1992], 667, AAS 85 [1993], 761, 814, 909, AAS 87 [1995], 1011, AAS 88 [1996], 861, AAS 91 [1999], 9, 67, 363-364, 582, 789, 885, 1067, AAS 92 [2000], 248, AAS 93 [2001], 28, AAS 95 [2003], 132, 655, AAS 96 [2004], 112, 267, 281, 399.

9 Cf. AAS 85 [1993], 43, AAS 87 [1995], 28, AAS 88 [1996], 180, AAS 90 [1998], 142, AAS 92 [2000], 623.

10 Cf. AAS 87 [1995], 481-482, AAS 88 [1996], 944, AAS 95 [2003], 250-251.

11 AAS 71 [1979], 1575, AAS 83 [1991], 850.

12 AAS 74 [1982], 290, AAS 75, I, [1983], 498.

13 AAS 81 [1989], 94, AAS 96 [2004], 484. 
la chiesa nelle tempeste postconciliari ${ }^{14}$. Annotiamo due riferimenti al 'relativismo totale', ${ }^{15}$ e alle espressioni come 'l'autodistruzione relativistica', 'relativismo imperante', 'teorie relativistiche', 'atteggiamento relativistico', 'clima relativistico' e 'mentalità relativistica'16.

Una menzione particolare richiedono brani delle tre encicliche di Giovanni Paolo II, in quanto vi troviamo simili trattazioni del relativismo, e cioè la Centesimus annus, la Veritatis splendor e la Evangelium vitae. Nella Centesimus annus (1991) registriamo un'occorrenza del termine relativismo in un discorso situato al livello politico con menzione della democrazia e del ruolo della maggioranza ${ }^{17}$. Nella Veritatis splendor (1993) l'occorrenza del termine relativismo è di otto volte ${ }^{18}$. In questo testo il papa pone l'accento sull'assenza della verità, legata alle posizioni relativiste nel sistema democratico ${ }^{19}$. La Evangelium vitae (1995), invece, parla tre volte di relativismo etico e una volta del relativismo totale, ma l'enciclica in sostanza ribadisce il discorso delle due encicliche già viste accentuando la difesa della sacralità della vita umana ${ }^{20}$. I brani dei tre documenti citati hanno in comune questi elementi: 1) essi riguardano il tema del relativismo, della democrazia e della verità, 2) il concetto vi viene approfondito e non soltanto riportato accanto ad altri termini, come nella maggior parte delle occorrenze viste, 3) i tre testi risalgono all'inizio degli anni novanta.

La seconda pubblicazione su cui abbiamo condotto la ricerca terminologica sono gli Insegnamenti di Giovanni Paolo II, più precisamente gli indici di questi. In questi ultimi il termine relativismo appare soltanto due volte: la prima nell'anno 1997, la seconda nel $1999^{21}$. Negli indici analitici dell'Enchiridion Vaticanum, che è la terza pubblicazio-

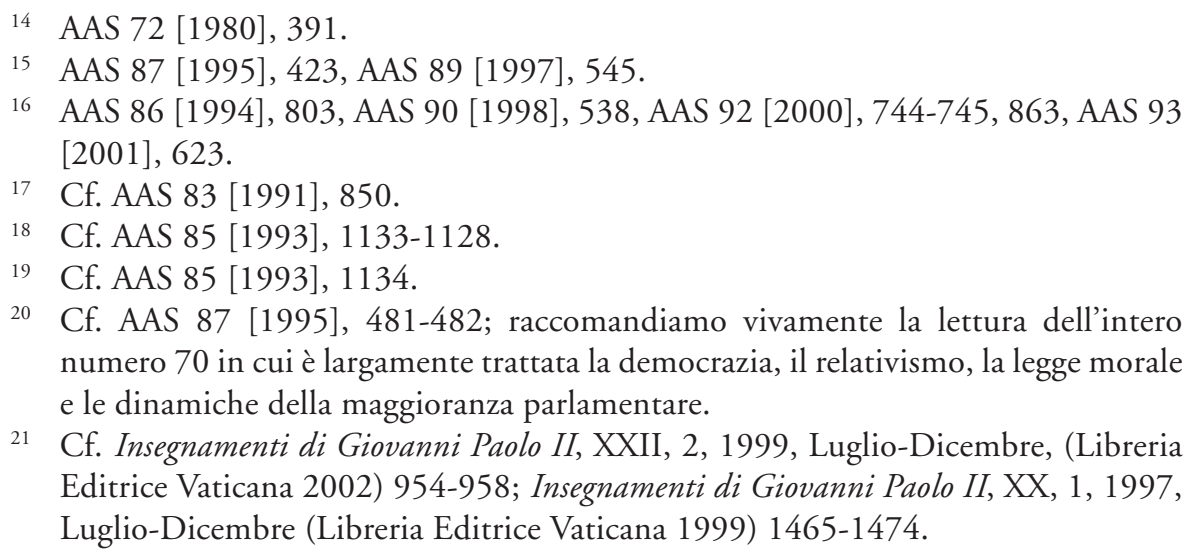
numero 70 in cui è largamente trattata la democrazia, il relativismo, la legge morale e le dinamiche della maggioranza parlamentare.

21 Cf. Insegnamenti di Giovanni Paolo II, XXII, 2, 1999, Luglio-Dicembre, (Libreria Editrice Vaticana 2002) 954-958; Insegnamenti di Giovanni Paolo II, XX, 1, 1997, Luglio-Dicembre (Libreria Editrice Vaticana 1999) 1465-1474. 
ne esaminata, il termine è ugualmente recepito molto tardi. A partire dall'inizio del pontificato fino all'anno duemila, esso appare soltanto tre volte. Negli anni successivi tali occorrenze sono più numerose ${ }^{22}$. È assai sorprendente che i curatori degli indici non diano importanza a questo concetto, ma forse tale omissione non è dovuta soltanto all'attenzione dei curatori. Più tardi cercheremo di riflettere su quest'omissione di riferimenti.

Abbiamo potuto osservare la complessità del discorso sul relativismo nel Magistero di Giovanni Paolo II, che si manifesta dai suoi molteplici discorsi, nei quali il termine assume svariate accezioni. Il relativismo è una mentalità, un atteggiamento, un paradigma, una teoria e filosofia che minaccia la convivenza stessa della società. In suo nome si commettono crimini e le sue dinamiche sono opposte a quelle delle persone con una convinzione per la verità. Quando guardiamo l'occorrenza del termine 'relativismo' lungo tutti gli anni del pontificato di Giovanni Paolo II scopriamo che essa comincia a crescere a partire dall'inizio degli anni novanta, con particolare accento sulla morale. Gli indici però recepiscono circa dieci anni più tardi la presenza di questo termine. Come se il relativismo nei testi di Giovanni Paolo II cominciasse ad avere l'importanza solo a partire dagli anni novanta; ma esso viene avvertito gradualmente e soltanto attorno l'anno duemila. Che cosa succede all'inizio degli anni novanta? A che cosa è dovuto tale aumento dell'attenzione verso il relativismo e la sua successiva sottolineatura nei testi presentati? Noi crediamo che la risposta si trovi in tre encicliche di Giovanni Paolo II, appena citate, e in alcuni articoli chiave di Joseph Ratzinger.

\section{Joseph RATZINGER E RELATIVISMo}

La nostra indagine sul relativismo nel pensiero di Joseph Ratzinger si restringe al periodo che va dalla metà degli anni settanta in poi. Abbiamo deciso di considerare il suo pensiero soltanto a partire da quegli

22 Il termine 'relativismo storicistico' è presente in Enchiridion Vaticanum 9, Documenti ufficiali della Santa Sede 1983-1985, (EDB, Bologna 1997) n. 1130, inoltre l'Enchiridion Vaticanum 13, 1991-1993, fa riferimento a Veritatis Splendor, e a Enchiridion Vaticanum, 17, Documenti ufficiali della Santa Sede 1998, (EDB, Bologna 2000) fa riferimento al termine presente in Fides et Ratio. Nel 2005 si pubblica l'Enchiridion Vaticanum, Supplementum 3, Documenti ufficiali della Santa Sede, Omissa, Indici Generali 1988-2001, che colma tale lacuna con nove riferimenti al termine 'relativismo'. 
anni perché è difficile scorgere nei suoi testi anteriori i temi che si relazioneranno successivamente con il relativismo. Joseph Ratzinger, negli scritti degli anni del concilio e in quelli postconciliari, si occupa dei temi strettamente teologico-dogmatici e intraecclesiali. E anche se in qualche titolo vi trovassimo l'indicazione ad un tema che potesse essere legato a quello del relativismo (dove successivamente tratterà temi come mondo, storia, sistemi politici, e così via), tale cenno indica sempre un trattamento teologico differente da quelli successivi in cui collocherà quello del relativismo ${ }^{23}$. Le sue successive trattazioni presentano un altro carattere, meno intra e più extraecclesiale. Ad ogni modo, anticipiamo che il discorso sulla fede sarà sempre presente, anche se a volte in maniera implicita, in tutte le trattazioni successive sul relativismo, quando Joseph Ratzinger dialogherà con interlocutori che non condividono il terreno della fede cristiana.

\subsection{Metà anni settanta - anni ottanta}

A partire dalla metà degli anni settanta Joseph Ratzinger comincia a dedicarsi ai temi etici e politici, arricchendo così il suo campo d'interesse che era prevalentemente teologico-dogmatico, specialmente ecclesiologico. Si può dire, con le sue parole, che egli cerca di uscire dal linguaggio interno della fede che è di tipo autoritativo e, quindi, di sviluppare un altro linguaggio fuori dal cerchio ristretto dei credenti ${ }^{24}$. Egli con toni allarmanti osserva la situazione politico-sociale del vecchio continente:

«La pluralità dei valori, che è legittima ed è europea, crescerà a vista d'occhio verso un pluralismo dal quale viene sempre più escluso ogni ancoraggio morale del diritto e ogni ancoraggio pubblico del sacro e del timore di Dio come valore anche collettivo. Interrogarsi su ciò

23 Cf. J. Ratzinger, Problemi e risultati del Concilio Vaticano II, (Queriniana, Brescia 1967); J. Ratzinger, "Salvezza e storia", in Storia e dogma, (Jaca Book, Milano 1971) 93-110 [orig.: J. Ratzinger, Heil und Geschichte, in Wort und Wahrheit 25 (1970), 3-14]; J. Ratzinger, C. Pauwels, Y. Congar, E. Schweitzer, A. Winklhofer, La chiesa ai nostri giorni, (Edizioni Paoline, Roma 1968) 7-26 [orig. in tedesco 1965]; J. Ratzinger, "Democratizzazione della Chiesa?", in J. Ratzinger - H. Maier, Democrazia nella Chiesa. Possibilità, limiti, pericoli, (Edizioni Paoline 1971) 5-56 [orig. in tedesco 1970]; J. Ratzinger, Fede e futuro, (Queriniana, Brescia 2005) [orig. in tedesco 1970]; J. RATZINGER, Il senso dell'esistenza cristiana, (Edizioni Paoline, Francavilla 1966). 
sembra a molti già un offesa alla tolleranza e alla società fondata solo sulla ragione $»^{25}$.

Simili osservazioni diventeranno sempre più elaborate e i temi in cui si articolerà il concetto del relativismo avranno a che fare proprio con l'analisi politico-sociale e religiosa dell'Europa, con la crisi dei valori e con la critica del sistema democratico. Le prime parole di un articolo sulla morale cristiana suonano così: "La crisi della fede, che incalza la cristianità in misura crescente, si manifesta sempre più chiaramente anche come una crisi di coscienza dei valori fondamentali della vita umana. Da un lato, quella è alimentata dalla crisi morale dell'umanità e, dall'altro, aggrava questultima a sua volta» ${ }^{26}$. In questo passo troviamo un primo cenno a ciò che può essere indicato con il termine relativismo, ma tale termine non è ancora utilizzato. Soltanto una quindicina d'anni dopo troverà il termine adatto per descrivere i sintomi della crisi morale: la liberazione dalla morale, il trasferimento del morale nel soggettivo, dissolvimento strisciante del matrimonio, degradazione della vita sessuale, lotta tra sessi e generazioni, l'aborto ${ }^{27}$. Il termine con cui egli descrive tali fenomeni è la "relativizzazione dell'ethos". Il teologo bavarese, quindi, in un passo estremamente complesso afferma:

«Le interpellanze critiche che si possono rivolgere al cristianesimo come forza politica, a partire dalla sua storia come dall'ora presente, le vorrei racchiudere in tre aspetti. [...] b) Un secondo elemento con cui il cristianesimo può influire esplosivamente su un ethos sostenitore dello stato è la sua negazione della giustizia che viene dalle opere e la conseguente misura della santità, come derivante unicamente dalla grazia. Può svilupparsi di qui una relativizzazione dellethos e un'incapacità del compromesso, la quale distrugge l'umiltà dell'imperfezione, di cui vive la collettività umana, come si è visto sopra» ${ }^{28}$.

25 J. Ratzinger, "L'Europa: Un'eredità vincolante per i cristiani”, in Perché siamo ancora nella Chiesa, (Rizzoli, Milano 2008) 163-183, qui 170 [l'articolo è un intervento in un convegno del 1979].

26 J. Ratzinger, "Magistero ecclesiastico-Fede-Morale", in J. Ratzinger, H. Schürmann, H. U. von Balthasar, Prospettive di morale cristiana, (Città Nuova, Roma 1986) 37-58, qui 37 [orig. in tedesco 1975].

27 Cf. J. Ratzinger, "Ordinamento cristiano nella democrazia pluralistica? Sulla irrinunciabilità del cristianesimo nel mondo moderno", in Chiesa, ecumenismo e politica. Nuovi saggi di ecclesiologia, (Edizioni Paoline, Cinisello Balsamo 1987) 190-206, qui 203.

28 J. Ratzinger, “Ordinamento cristiano...”, 198 (il corsivo è nostro). 
Il concetto di relativismo è qui legato al ruolo dello stato nell'esecuzione del suo potere. Lo stato può relativizzare, cioè destabilizzare l'ethos: "Il vero pericolo del nostro tempo, il centro della nostra crisi culturale è la destabilizzazione dell'ethos, la quale si fonda sul fatto che noi non possiamo più comprendere la ragione della dimensione morale ed abbiamo ridotto la ragione al calcolo esatto» ${ }^{29}$. In queste citazioni possiamo notare sia come Joseph Ratzinger gradualmente descrive e cerca di definire una complessa realtà con il termine relativismo, sia in che contesto il termine relativismo viene sviluppato. I suoi nuovi interessi sono: la democrazia, l'etica e lo stato, qui rappresentato dal sistema democratico pluralista. La critica di questa forma statale viene legata, inoltre, al tema della verità di fede. Il cardinale, due volte nello stesso articolo, paragona il pantheon romano con il sistema democratico. Oggi, come anticamente, il cristianesimo non deve entrare nel pantheon, perché la sua pretesa della verità di fede sia manifesta: "Ma questo ripiegamento nel privato, questo inserimento nel pantheon di tutti i possibili sistemi di valore, è in contraddizione con la pretesa di verità della fede, che come tale è una pretesa pubblica» ${ }^{30}$. Nel pantheon sono presenti tutte le verità religiose, tutte sono poste sullo stesso piano, tutte sono così diventate relative. Ma la il messaggio del cristianesimo non può essere relativizzato perché pretende di possedere la verità. E quella verità è la verità totale, cioè assoluta ${ }^{31}$.

Un importante articolo-relazione, che come vedremo successivamente, avrà una certa relazione con il relativismo, anche se il termine relativismo ancora non vi appare, porta la data 1989. Il cardinale avverte il

29 J. RatZinger, “Ordinamento cristiano...”, 202.

30 J. Ratzinger, "Ordinamento cristiano...”, 204, cf. 199: «La fede cristiana ha infranto l'antica idea di tolleranza, nel suo contenuto, per la ragione che esso non si lasciava immettere nel pantheon, che costituiva lo spazio della tolleranza religiosa, nello scambio e nel vicendevole riconoscimento degli dèi. Giuridicamente, questa fede non poteva giungere a beneficiare di questa tolleranza perché si rifiutava di farsi collocare nell'ambito del diritto privato, in cui aveva il suo luogo qualsiasi spontanea formazione religiosa. Questo inserimento nel diritto privato no era possibile per la fede cristiana perché il diritto pubblico era il diritto degli dèi. Il monoteismo cristiano non poteva ritirarsi in un diritto privato: ciò l'avrebbe eliminato come monoteismo nella sua pretesa di verità»; cf. J. RATZINGER, "Il problema dell'assolutezza del camino cristiano", in Perché siamo ancora...., 37-55, qui 50-51 [l'articolo è un intervento in un convegno del 1966]; cf. J. Ratzinger, "L'Europa: Un'eredità...", 180-181.

31 Sull'accessibilità dell'assoluto nella fede cristiana in contrasto con il carattere politeistico moderno, cf. J. RatZInger, "Il problema dell'assolutezza...”, 46. 
cambiamento del paradigma nella società. Egli nell'articolo analizza la situazione della chiesa nel mondo, più precisamente una certa contestazione all'interno della chiesa e le origini di tale disagio: "Considerando queste motivazioni della contestazione, che ritornano continuamente pur sotto forme diverse, diventa chiaro che ciò che è in causa in questo canone, apparentemente così circoscritto, è in realtà un coerente nuovo orientamento

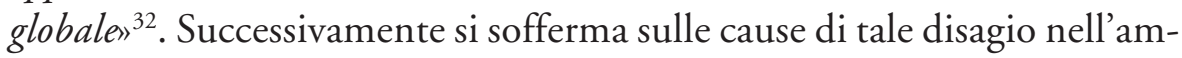
bito teologico. A noi interessa piuttosto la conclusione dell'articolo:

«[...] volevo solo cercare di esaminare, al di là dei singoli problemi che riemergono continuamente, le motivazioni più profonde dalle quali nascono in forme sempre mutevoli le singoli difficoltà. Soltanto se noi impariamo a comprendere quella sensibilità fondamentale dell'esistenza moderna [...] Solo allora potremo rendere visibile la fede come l'alternativa che il mondo attende dopo il fallimento dell'esperimento liberalistico e quello marxista ${ }^{33}$.

Dalle ultime righe abbiamo appreso l'arrivo di un imminente cambiamento del paradigma nella società e che i problemi passati erano il liberalismo e marxismo. Quale sarà, però, il nuovo paradigma della società e quale l'elemento dominante nelle sue radici? Abbiamo visto che nel periodo appena esaminato viene inizialmente osservata una generale crisi morale -europea- che è la crisi dei valori, legata al pluralismo, e che è per di più negli scritti di Joseph Ratzinger un tema nuovo. Da qui rileviamo una prospettiva del discorso che si situa al livello politico e che vedremo con il corso degli anni approfondito sempre di più. Il fulcro della crisi viene individuato nel funzionamento del sistema democratico. Inoltre, osserviamo la ricerca della chiarezza terminologica nel racchiudere i fenomeni di questa crisi sotto un denominatore comune. Verso la fine degli anni ottanta Joseph Ratzinger trova l'espressione la 'relativizzazione dell'ethos'. Con ciò constatiamo che siamo ad un passo dalla chiarezza terminologica definitiva, ma in questo periodo essa non è ancora completamente raggiunta.

32 J. Ratzinger, "Difficoltà di fronte alla fede oggi in Europa", in Il Regno Attualità 36/264 (1989), 543-546, qui 543.

33 J. Ratzinger, "Difficoltà di fronte...", 546. 


\subsection{Anni novanta e duemila}

Spostandosi agli anni novanta i temi appartenenti al concetto del relativismo negli scritti di Joseph Ratzinger diventano più specifici e più approfonditi, e mentre viene svolta un'indagine culturale e politica del tempo, non si tralascia la relazione o le possibili conseguenze di ciò per la comunità ecclesiale. A volte, quindi, i temi strettamente teologici e filosofici, con chiara prospettiva della fede e della morale cattolica, vengono trattati e messi a confronto con alcuni aspetti politici della società. In questa sezione riportiamo i brani in cui appaiono per la prima volta nei testi di Joseph Ratzinger certi temi associati al relativismo, e che egli successivamente espone in varie opere. Curiosamente, grazie ad Albert Einstein il cardinale Joseph Ratzinger arriva a descrivere il contesto relativistico etico:

«La teoria della relatività formulata da Einstein concerne, come tale, il mondo fisico. A me sembra però che possa descrivere adeguatamente anche la situazione del mondo spirituale del nostro tempo. La teoria della relatività afferma che all'interno dell'universo non si dà nessun sistema fisso di riferimento. [...] In un mondo senza punti fissi di riferimento non ci sono più direzioni. Ciò cui guardiamo come ad un orientamento non si basa su un criterio vero in se stesso, ma su una nostra decisione, ultimamente su considerazioni di utilità. In un simile contesto "relativistico" un'etica teleologica o consequenzialistica diventa ultimamente nichilistica, anche se non ne ha la percezione. E quanto in questa concezione della realtà viene chiamato "coscienza», ad una più profonda riflessione, si mostra essere un modo eufemistico per dire che non c'è nessuna coscienza in senso proprio, cioè nessun «con-sapere» con la verità. Ognuno determina da solo i propri criteri e, nell'universale relatività, nessuno può neppure essere d'aiuto a un altro in questo campo, e meno ancora prescrivergli qualche cosa ${ }^{34}$.

Da questa sua riflessione sul relativismo apprendiamo che in esso non si danno più i punti di riferimento, e senza questi non ci sono direzioni. Conseguenza di ciò è l'impossibilità di etiche con orientamenti precisi,

34 J. Ratzinger, "Coscienza e verità", in La Chiesa. Una comunità sempre in cammino, (Edizioni Paoline 1991) 126 (il corsivo è nostro); lo stesso testo è stato presentato in inglese a Dallas (1991), cf. http://www.ewtn.com/library/curia/ratzcons. htm (19/04/2013). Per la concezione di coscienza in liberalismo, cf. J. Ratzinger, "Coscienza e verità", 117, 121. 
cioè con punti e direzioni, e in ultima analisi anche la perdita di coscienza. In questo brano possiamo osservare come Joseph Ratzinger sviluppa l'argomento del relativismo, come esso matura, e a che cosa, o a chi, è dovuta la sua riflessione. Lo stesso processo di maturazione terminologica si mostra qui ancor più chiaramente. Il termine relativismo non compare, mentre troviamo le espressioni "contesto relativistico" e "universale relatività". Ad ogni modo, nello stesso anno esce un'altra pubblicazione in cui la 'relatività' diventa un -ismo, vale a dire il 'relativismo':

"Considerando sinteticamente le forze di cui abbiamo parlato finora, possiamo ricondurle a due indirizzi fondamentali: il relativismo e la fede. Il relativismo si allea facilmente col positivismo; di quest'ultimo esso costituisce, in realtà, il fondamento filosofico vero e proprio. Che in alcune situazioni un pizzico di relativismo e un poco di scetticismo possano giovare, non intendiamo metterlo in discussione. Ma il relativismo risulta totalmente inadeguato a far da comune fondamento su cui poter vivere. Se infatti esso si concepisce e si attua in modo coerente -senza appoggiarlo di nascosto ad un'ultima certezza di fede- $\mathrm{o}$ finisce per ridursi a nichilismo, o erige una mentalità di tipo positivista a potere che tutto dispone, sfociando nuovamente così in concezioni totalitarie. Ma se lo scetticismo e il relativismo, nonostante la loro parziale utilità, non offrono un itinerario globalmente soddisfacente, quali altre possibilità restano allora? Non siamo ancora una volta sollecitati a volgere lo sguardo all'autosuperamento dell'uomo, all'itinerario di fede nel Dio vivente? ${ }^{35}$.

Anche qui, come negli altri scritti visti in precedenza, Joseph Ratzinger non tratta il relativismo in maniera isolata, ma lo pone accanto alla trattazione sulla fede. Nella sua riflessione viene riconosciuto che il relativismo e lo scetticismo, considerati come accidenti, sono parzialmente utili, ma nonostante ciò, il relativismo, se è tale, non può appoggiarsi su alcuna certezza, e così si svela la sua insostenibilità teorica. Il teologo Joseph Ratzinger, allora, dopo che ha svelato la nullità di queste posizioni, c'indicherà con due questioni retoriche la strada da percorrere: la via della fede.

A nostro avviso, il più importante articolo sul relativismo di Joseph Ratzinger, in cui appariranno tutti i temi ad esso legati, anche nei suoi

35 J. Ratzinger, "Itinerari della fede tra i rivolgimenti del tempo presente", in Svolta per l'Europa? Chiesa e modernità nell'Europa dei rivolgimenti, (Edizioni Paoline, Cinisello Balsamo 1992) 65-89, qui 82 (il corsivo è nostro) [orig. in tedesco 1991]. 
scritti posteriori, è stato presentato a Bratislava in una conferenza con i vescovi slovacchi nell'anno $1992^{36}$. Quindi, per capire la sostanza del suo pensiero maturo al riguardo, basterebbe la lettura di questo testo. Analizziamo i punti salienti della prima parte del testo, dedicata al relativismo. Nella sezione corrispondente al primo titolo dell'articolo 'Il relativismo come necessario presupposto della democrazia?' si riporta la convinzione comune che la democrazia è, dopo il crollo dei regimi totalitari "l'unico sistema di governo adeguato ai nostri tempi», in cui la partecipazione di tutti al potere è l'espressione della libertà, tramite il potere delegato agli altri ${ }^{37}$. Tale libertà per non autodistruggersi ha bisogno di criteri, limiti e contenuto. Questi ultimi diventano la salvaguardia dei diritti umani, la "garanzia del progresso della società tutta e del bene dei singoli individui ${ }^{38}$. Successivamente rileva tre nozioni che sono emerse, libertà, diritto e bene, e in riferimento allo Stato e afferma:

«L'attenzione alla libertà di ciascun individuo ci sembra oggi consistere essenzialmente, ed in linea di principio, nel fatto che lo Stato non pretenda di risolvere il problema della verità: la verità -dunque anche la verità circa il bene- non appare conoscibile nella sfera sociale. Essa è piuttosto controversa. [...] Essa non è un bene "pubblico", bensì soltanto "privato" o, tutt'al più, un bene "di parte", non universale. In altri termini, la nozione moderna di democrazia sembra essere indissolubilmente legata all'opzione relativista; il relativismo appare come l'unica vera e propria garanzia della libertà e proprio il suo nucleo essenziale: la libertà religiosa e la coscienza» ${ }^{39}$.

In seguito continua a domandarsi se nella democrazia c'è anche un nucleo non-relativistico e lo trova nei diritti umani, che presentano un contenuto veritativo -verità- ma alcuni preferiscono parlare di valore. Rispetto a ciò ci sono due possibili dibattute soluzioni politiche: l'opzione relativista con il gioco di maggioranze e la concezione metafisica con il primato della verità sulla politica ${ }^{40}$. Successivamente, ci propone

36 J. RATZINGER, "Sul significato dei valori religiosi e morali nella società pluralistica", in L'elogio della coscienza. La verità interroga il cuore, (Edizioni Cantagalli, 2009) 49-76.

37 Cf. J. Ratzinger, "Sul significato dei valori...”, 49.

38 Cf. J. Ratzinger, "Sul significato dei valori...”, 50-51.

39 Cf. J. Ratzinger, "Sul significato dei valori...", 51-52 (il corsivo è nostro).

40 Cf. J. Ratzinger, "Sul significato dei valori...”, 52-55. Queste posizioni sono spiegate dettagliatamente sulle pagine 60-66. Per approfondire il nesso democraziadiritti fondamentali-relativismo cf. J. RATZINGER, "Il problema delle minacce alla 
una figura relativista per eccellenza, quella cioè di Ponzio Pilato, ritratta seguendo studi di Hans Kelsen e Heinrich Schlier. Pilato non aspetta da Gesù la risposta alla domanda Che cos’è la verità?, ma si gira verso la folla, perché in essa la maggioranza decida sulla sorte di Gesù ${ }^{41}$. Tutto ciò porta il cardinale ad interrogarsi sul fine, sulla funzione e sull'essenza dello Stato. In quest'ultimo articolo si constata la fine dei regimi totalitari e il cambiamento verso la democrazia che viene associata al relativismo. Quindi, mentre in un articolo del 1989, che abbiamo esaminato in precedenza, si accerta il fallimento del liberalismo e del marxismo e l'emergere del nuovo paradigma, senza però nominarlo da vicino, qui troviamo già definito questo nuovo paradigma: la democrazia e il relativismo. Tale interpretazione è confermata da altri due articoli, in cui troviamo di nuovo riferimenti al marxismo e alla caduta dei regimi nel cosiddetto Est europeo. Si tratta di un articolo sui recenti cambiamenti europei del 1991 e di una conferenza della Congregazione per la Dottrina della fede, tenutasi a Guadalajara nel $1996^{42}$. Nel primo testo riflette sulla caduta dei regimi europei:

«La fuoriuscita da un'ideologia, un tempo ragione di vita, può molto facilmente capovolgersi anche in nichilismo, e ciò sarebbe allora veramente il dominio dei sette spiriti più malvagi. Chi può nascondersi che il relativismo, al quale noi tutti oggi siamo esposti, produca

vita umana", in La via della fede..., 107-123, qui 110-111; J. RATZINGER, "Ragione e fede. Scambio reciproco per un'etica comune", in J. Habermas - J. Ratzinger, Ragione e fede in dialogo, G. BosetTi, (a cura di), (Marsilio, Venezia 2005), 65-81, qui 65-68.

41 Cf. J. Ratzinger, "Sul significato dei valori...”, 55-57. Questo emblematico esempio di Ponzio Pilato viene ripetuto in Natura e compito della teologia. Il teologo nella disputa contemporanea. Storia e dogma, (Jaca Book, Milano 1993) 40; J. RATZINGER, "Libertà e verità", in La via della fede. Saggi sull'etica cristiana nell'epoca presente, (Edizioni Ares, Milano 1996) 13-36, qui 13; BenedeTto XVI: Luce del mondo. Il papa, la Chiesa e i segni dei tempi, Una conversazione con Peter Seewaald, (Libreria Editrice Vaticana, 2010) 81; La Via Crucis 2007 (Colosseo, Roma), Stazione V; J. RatZINGER, "Fede, religione e cultura", in Fede, Verità, Tolleranza. Il Cristianesimo e le religioni del mondo, (Edizioni Cantagalli, Siena 2003) 57-82, qui 74-75; J. RatZinger, "Verità, tolleranza, libertà", in Fede, Verità, Tolleranza..., 223-275, qui 245-246.

42 J. Ratzinger, "La fede e la teologia ai giorni nostri”, in Civiltà Cattolica IV (1996), 477-490. Questo articolo è stampato altre due volte: cf. J. RaTZINGER, "Relativismo problema della fede”, in Il Regno-Documenti 784/1 (1997) 51-56; e J. RATZINGER, "Le nuove problematiche avviatesi negli anni novanta sulla situazione della fede e della teologia oggi”, in Fede, Verità, Tolleranza..., 119-143. 
una crescente inclinazione al nichilismo? L'interrogativo si fa così stringente: con quali contenuti possiamo colmare il vuoto spirituale, creatosi con il fallimento dell'esperimento marxista? Su quali fondamenta spirituali possiamo costruire un comune futuro, in cui Est e Ovest si leghino in una nuova (esperienza di) unità, ma anche Nord e Sud trovino un cammino comune?» ${ }^{43}$.

I paesi dell'Est europeo sono in questi anni in transizione da un sistema politico all'altro. La preoccupazione che si rileva è quella di trovare nuovi punti di partenza dopo la fine dell'ideologia marxista e di costruire un comune futuro europeo. Il relativismo crescente sembra così essere la nuova preoccupazione di primaria importanza. Nella prima parte del secondo articolo Joseph Ratzinger associa, tramite il marxismo, la teologia della liberazione alla caduta dei regimi dell'Est. Mostrata la caduta dei regimi si mostra l'insufficienza e gli orrori del marxismo, considerato passato, ed un occhio attento scopre il motivo di quelle righe. Adesso «il relativismo è diventato perciò effettivamente il problema fondamentale della fede nei nostri giorni. [...] si presenta inoltre come la base filosofica della democrazia» ${ }^{44}$. In seguito, gran parte dell'articolo è dedicata alla contestazione delle teorie di J. Hick e di Knitter, che rappresentano il relativismo sul piano teologico nel campo del pluralismo religioso. La conferma di tale interpretazione della società contemporanea, con il rilievo della caduta dei regimi dell'Est e del relativismo come il suo gran pericolo, si trova anche in un'intervista a mons. Tarcisio Bertone, collaboratore di Joseph Ratzinger durante quell'incontro della Congregazione per la Dottrina della fede ${ }^{45}$.

Anche in un'intervista del 1994 Joseph Ratzinger conferma le tesi relative alla società presente, rispondendo al giornalista che gli domanda quale sia l'interpretazione che prevale nella Veritatis Splendor: "[...] lei ha sottolineato la risposta in essa contenuta rispetto alle tendenze culturali di tipo soggettivistico e relativistico. L'ottica intraecclesiale ha invece sottolineato l'elemento precettivo sugli squilibri della recente etica teologica». Il già prefetto Ratzinger risponde, confermando queste tendenze culturali:

43 J. Ratzinger, "Svolta per L'Europa”, in Svolta per l'Europa? Chiesa e modernità nell'Europa dei rivolgimenti, (Edizioni Paoline, Cinisello Balsamo 1992) 118-145, qui 120 (il corsivo è nostro) [orig. in tedesco 1991].

44 J. RATZINGER, “Svolta per L'Europa”, 478.

45 Cf. Intervista a mons. T. Bertone sull'incontro di Guadalajara (Messico): “Teologie e sfide pastorali", in Il Regno-Documenti 14 (1996) 403ss. 
«Non avrei mai detto quel che ho detto alla stampa se non fossi convinto che l'ottica dell'enciclica è appunto non quella di fomentare discussioni intraecclesiali, condotte da una teologia che si chiude in se stessa, sulle proprie controversie, ma la volontà di parlare all'uomo di oggi. È un grande documento di dialogo con il mondo e le sue pesanti sofferenze, nutrito di fede cristiana. Mi sembra innegabile che il mondo stia attraversando una crisi epocale, che coinvolge i valori portanti e attraversa anche le grandi religioni. È un'evidenza incontrovertibile che abbiamo bisogno di valori etici» ${ }^{46}$.

Nei testi posteriori troviamo ulteriori approfondimenti dei discorsi già visti in precedenza. In uno di questi appare interessante la indicazione che il relativismo ha a che fare con le istanze della terra, anche se successivamente non si comprende questo iniziale accenno alla terra, perché semplicemente viene omesso successivamente ${ }^{47}$. Però, sulla base dei discorsi precedenti, cerchiamo di dare la nostra spiegazione del relativismo legato alla terra. Sembra che qui si tratta, in fondo, non tanto delle istanze terrene, quanto piuttosto della negazione delle istanze dei cieli, quindi quelle escatologiche. Relativismo quindi nega la stessa possibilità della salvezza dell'uomo, e con ciò la sua natura orientata in maniera trascendente. E forse questa critica riguarda anche la teologia della liberazione che pone le speranze nella liberazione dall'oppressione terrena, qui ed ora. Di nuovo, come nell'immagine del pantheon, anche qui viene fatto ricorso alla democrazia. Ciò che oggi non è "democratico" viene rifiutato e contestato. E la verità ha sempre pretese assolute, quindi non democratiche, non di parte, non appartiene a un gruppo e si impone. Nelle dinamiche della democrazia non si comprende più la verità, anzi in essa si minaccia l'identità cristiana: quella verità che i cristiani devono proclamare. La democrazia, in questo modo, è propria dell'uomo moderno:

"Poi citerei ancora altri due temi, che hanno a loro volta a che fare con la terra. Il primo è quello dell'ecologia. [...] L'altro tema, a cui

46 J. Ratzinger, "Modernità atea, religiosità post-moderna", in Il Regno Attualità 721, 4 (1994) 65-70, qui 66.

47 La spiegazione più probabile potrebbe essere che si tratti di un errore nella redazione del testo, di qualche riga omessa dalla stampa; annotiamo che questo è l'unico riferimento del relativismo in relazione con la terra, negli scritti di Joseph Ratzinger. 
desidero far riferimento, è la corrente relativistica, che è divenuta molto forte. Essa ha origini diverse. Da una parte, dire: «abbiamo la verità», appare all'uomo moderno come qualcosa di non democratico, intollerante e anche inconciliabile con il necessario scetticismo dello scienziato, dall'altra si dice che la verità non c'è o che se ne hanno solo dei frammenti. Proprio a partire da una concezione della democrazia della vita e dall'idea di tolleranza che ne deriva è divenuta molto urgente la domanda se ci sia lecito continuare ad affermare la nostra autocoscienza cristiana ${ }^{48}$.

Accertiamo così che due colonne della critica del relativismo di Joseph Ratzinger, stabilmente presenti in tutte le sue riflessioni, sono il discorso sulla fede, cioè la preoccupazione di dare un orientamento al singolo cristiano, ed il discorso sulle tendenze della società contemporanea. Con un cenno nella nota a piè di pagina, riportiamo la presenza degli stessi temi già apparsi e riguardanti il relativismo in maniera implicita: la tolleranza, la libertà, la legislazione, la soggettività, la coscienza e la moralità ${ }^{49}$. Indichiamo lo sviluppo dei temi come la democrazia, la verità, lo scetticismo e la moralità ${ }^{50}$; citiamo inoltre un discorso sulla verità e la sua sostituzione con il consenso ${ }^{51}$. Altri problemi su cui Joseph Ratzinger teorizza in relazione alla morale sono: l'oggettività, la soggettività, la coscienza, la riduzione della morale al soggettivo, ma senza il riferimento al relativismo (forse perché la conferenza è del 1987) ${ }^{52}$. Alla "prigione relativistica" è accennato in una conferenza in San Giovanni in Laterano nel $1999^{53}$. Annotiamo anche un altro riferimento al tema della verità e

48 J. Ratzinger, Il sale della terra. Cristianesimo e la Chiesa cattolica nella svolta del millenio, (San Paolo, Cinisello Balsamo 1997) 153-154 (il corsivo è nostro) [orig. in tedesco 1996].

49 Cf. J. RAtzinger, "Il problema delle minacce alla vita umana”, 112, 114-115, 116.

50 Cf. J. RATZinger, "Libertà e verità", 22-23.

51 Cf. J. RatZinger, "Libertà e verità", 31.

52 Cf. J. Ratzinger, "Il compito del vescovo e del teologo innanzi ai problemi morali del nostro tempo", in La via della fede, 55-77, qui 57. Per la trattazione degli stessi temi, senza il riferimento esplicito al relativismo cf. "Sulla dignità della persona", in La via della fede, op. cit., 35-48.

53 Cf. J. Ratzinger, "Conferenza svolta in San Giovanni in Laterano", in AA.VV., Per una lettura dell'enciclica Fides et ratio, (Città del Vaticano 1999) 245-259, qui 254: "Certamente la fede non può stringere legami con filosofie, che escludono la questione della verità, ma certamente lo può fare con quei movimenti, che cercano di uscire dalla prigione relativistica. Certamente essa non può riallacciarsi immediatamente alle antiche religioni $[\ldots]$ ». 
della maggioranza, ed al relativismo delle culture con l'universalismo del cristianesimo ${ }^{54}$. Vale la pena di ricordarsi dell'implicito riferimento a ciò che Joseph Ratzinger più tardi chiamerà la "dittatura del relativismo" in un'intervista del 2001. Egli nel paragrafo intitolato 'Nuovi rischi per la fede' risponde alla domanda sul rischio del cristianesimo in una società ostile ad esso:

«[...] io credo che questo sia un pericolo reale. Certo, i cristiani non sarebbero apertamente perseguitati; sarebbe una cosa troppo di altri tempi, poco elegante. No, bisogna essere tolleranti, aperti a tutto. Ma poi ci sono cose che vanno decisamente escluse, etichettate come fondamentaliste, anche laddove si può invece trattare di vera fede. Penso che si possa creare una situazione in cui occorrerà opporre resistenza, resistenza a una dittatura di tolleranza apparente che vuole mettere fuori gioco lo scandalo della fede liquidandolo come intollerante. Qui verrebbe allora davvero alla ribalta l'intolleranza dei 'tolleranti' "s5.

In questo testo, accanto alla trattazione sulla tolleranza e sul pericolo per la fede, viene usata la parola "dittatura". Joseph Ratzinger retoricamente e analogicamente sfrutta la forza di questo termine, che richiama i regimi dell'Est europeo, già varie volte accennati. Evidentemente, perché vi vede le analogie e pericoli per la fede sotto tale dominio. Precedentemente si è accennato al legame tra la tolleranza ed il relativismo, ed è quindi facile concludere che qui è proprio del relativismo che sta parlando. Successivamente riaffermerà varie volte questo discorso, soprattutto nella Missa pro eligendo ${ }^{56}$. Qualche anno più tardi il cardinale pubblica un libro con Marcello Pera, in cui è stampata una sua lettera e alcune osservazioni sul relativismo, già affrontate in altri testi ${ }^{57}$.

54 Cf. J. RATZinger, "Fede, religione e cultura”, 74-76.

55 J. Ratzinger, Dio e il mondo. Essere cristiani nel nuovo millennio, Joseph Ratzinger in colloquio con Peter Seewald, (Cinisello Balsamo 2001) 415; per approfondire il nesso relativismo-democrazia-tolleranza cf. J. RATZINGER, “Non uccidere': Introduzione al terzo capitolo dell' 'Evangelium vitae', in La via della fede, 125-132, qui $130-131$.

56 AAS 97 [2005], 687: «Mentre il relativismo, cioè il lasciarsi portare «qua e là da qualsiasi vento di dottrina», appare come l'unico atteggiamento all'altezza dei tempi odierni. Si va costituendo una dittatura del relativismo che non riconosce nulla come definitivo e che lascia come ultima misura solo il proprio io e le sue voglie» (il corsivo è nostro).

57 J. Ratzinger, "Lettera a Marcello Pera", in M. Pera - J. Ratzinger, Senza radici. Europa, relativismo, cristianesimo, Islam, (Mondadori, Milano 2004) 97-122, qui 
I suoi libri scritti a quattro mani con quest'interlocutore laico a ateo sono marcati per gli approfondimenti del discorso politico sulla società attuale. Nell'ultimo libro, che chiude il periodo del suo cardinalato, di nuovo riappaiono gli stessi temi che oramai gli sono propri e che sono giunti ad essere maturi; essi rimarranno legati al relativismo anche nelle trattazioni successive ${ }^{58}$. Da quello che vi leggiamo sembra che il tema preferito e specifico di Benedetto al riguardo è la critica della morale e il suo ripiego nel soggettivo, la situazione e la critica della cultura europea, la tolleranza, le sfere decisive della vita pubblica e della dignità umana ${ }^{59}$.

Infine è opportuno soffermarci su alcune brevi trattazioni del relativismo nei documenti della Congregazione per la Dottrina della fede che portano la firma di Joseph Ratzinger. In un testo del 1984 troviamo un accenno al "vero significato dell'etica in cui non può essere relativizzata la distinzione tra il bene e il males ${ }^{60}$. L'anno seguente, invece, il cardinale avverte negli scritti di Leonardo Boff un relativismo di tipo ecclesiologi$\mathrm{co}^{61}$. Un documento in cui è in dettaglio trattato il tema del relativismo è intitolato Nota dottrinale circa alcune questioni riguardanti l'impegno e il comportamento dei cattolici nella vita politica ${ }^{62}$. Questo documento, che non riportiamo per la lunghezza del testo, ma che è degno di essere letto, si sofferma sui pericoli del relativismo per la democrazia. Notia-

116: «Negli ultimi tempi mi capita di notare sempre di più che il relativismo-quanto più diventa la forma di pensiero generalmente accettata- tende all'intolleranza, trasformandosi in un nuovo dogmatismo. La political correctness, la cui pressione onnipresente Lei ha evidenziato, vorrebbe erigere il regno di un solo modo di pensare e parlare. Il suo relativismo apparentemente la innalza più in alto di tutte le grandi vette del pensiero finora raggiunte; soltanto così si dovrebbe ancora pensare e parlare se si vuole essere all'altezza del presente. Mentre la fedeltà ai valori tradizionali e alle conoscenze che li sostengono viene bollata come intolleranza e lo standard relativistico viene elevato a obbligo. Mi sembra molto importante contrapporsi a questa costrizione di un nuovo pseudoilluminismo che minaccia la libertà di pensiero e anche la libertà di religione» (il corsivo è nostro); cf. 117: «[...] il relativismo comincia a prendere piede come una sorta di nuova «confessione», che pone limiti alle convinzioni religiose e cerca di sottoporle tutte al super-dogma del relativismo".

58 J. Ratzinger, Europa di Benedetto nella crisi delle culture, (Libreria Editrice Vaticana ed Edizioni Cantagalli, Roma-Siena, Maggio 2005).

59 J. RATZINGER, Europa di Benedetto..., 54-55; cf. 30, 52-53.

60 Cf. AAS 76 [1984], 907.

61 Cf. AAS 77 [1985], 759.

62 Cf. AAS 96 [2004], 361-362, 370. 
mo in esso un riferimento singolare. Nel testo si dice che il pluralismo etico è la condizione della democrazia e si citano la Centesimus annus e la Veritatis splendor, ma in esse non vi è presente tale riferimento; il riferimento al relativismo etico come condizione della democrazia è presente invece in Evangelium vitae, però quest'enciclica non è citata. Un errore di stampa, un poco di imprecisione, di confusione, oppure forse, la complementarietà tra il relativismo e il pluralismo?

Riassumendo questo periodo constatiamo che proprio negli inizi degli anni novanta, Joseph Ratzinger matura e definisce le sue posizioni, cioè la sua critica nei riguardi del relativismo. Egli negli scritti datati in questo periodo non abbandona più il termine relativismo, usa cioè soltanto questo termine per definire un certo elemento socio-politico osservato e dominante, e vi lega svariati temi. Dobbiamo dire che egli, dalla sua posizione di difensore della fede, dà un avvertimento ai cristiani sulla presenza del relativismo in vari strati della società, chiarendo cosa esso comporta e quali sono le sue conseguenze. Nei brani che abbiamo presentato, abbiamo rilevato due livelli di discorsi in cui situa il tema: uno è il dialogo politico sulla società, mentre l'altro è il discorso sulla fede per i cristiani. Essi si presentano a volte intrecciati, ed è il carattere del testo che permette di riconoscere quale dei due livelli predomina. Occorre domandarsi se Joseph Ratzinger concepisce fin dall'inizio il relativismo come relativismo etico. Osservando le trattazioni sul relativismo possiamo rispondere positivamente perché, in fondo, il relativismo riguarda il soggetto, il suo atteggiamento e le sue scelte, anche se l'aggettivo "etico" o "morale" appare nei testi poche volte. In sostanza, la critica che Joseph Ratzinger rivolge al relativismo è specificamente etica.

\subsection{Il pontificato di Benedetto XVI.}

Ricordiamo che le diverse accezioni del relativismo nel pontificato di Benedetto XVI saranno evidenziate considerando tre pubblicazioni magisteriali, come nel caso del Magistero precedente. Il numero di riferimenti al relativismo è circa settanta in sei anni e mezzo del suo papato, e il termine appare nella maggioranza dei casi senza un'ulteriore specificazione. Quando lo riterremo opportuno annoteremo le differenze o similitudini nell'uso del termine in comparazione al pontificato di Giovanni Paolo II. 
1) Il termine relativismo appare nella maggior parte dei casi elencato accanto ad altri termini con i quali è caratterizzata la società contemporanea. Abbiamo già riscontrato tale uso del termine nel pensiero del pontefice precedente. Quindi, il relativismo è considerato accanto alla secolarizzazione, all'indifferenza religiosa, al soggettivismo, all'individualismo estremo, all'utilitarismo, al nichilismo, al positivismo giuridico, al pragmatismo, al agnosticismo, al laicismo (una novità), al materialismo economico, al sincretismo, all'edonismo ed alla globalizzazione ${ }^{63}$.

2) Benedetto XVI diverse volte avverte il rischio del relativismo e del sincretismo nel dialogo interreligioso o ecumenico, spiegando inoltre che la famosa preghiera ad Assisi non è ricaduta nel relativismo ${ }^{64}$. Anche questo tipo di riferimento era già presente nel pontificato precedente.

3) Benedetto XVI percepisce i pericoli del relativismo per la famiglia e il matrimonio ${ }^{65}$. Con ciò annotiamo una novità nel riferimento del relativismo: nel magistero di Giovanni Paolo II non troveremo tale associazione del tema del relativismo alla famiglia e al matrimonio.

4) La difficoltà dell'educazione e della ricerca accademica, quando il sapere è frammentato: tutto ciò è esposto al rischio del relativismo. Un numero considerevole di riferimenti viene fatto riguardo alla verità: si nega la possibilità di attingere la verità, si disprezza la verità ${ }^{66}$. Constatiamo che tale discorso è proprio di Benedetto XVI, infatti in Giovanni Paolo II, manca tale associazione del tema del relativismo all'educazione e al sapere.

5 Benedetto XVI in vari discorsi cerca di spiegare più ampiamente che cos'è il relativismo; non solamente lo menziona o lo elenca tra

63 Cf. AAS 97 [2005], 687, 990, 1048, AAS 98 [2006], 38, 133, 807, AAS 99 [2007], 25, 87, 294, 427, 455, 567, 593, AAS 100 [2008], 76, 106, 168, 372, AAS 101 [2009], 847, 866, 907, AAS 102 [2010], 84, 108, 349, 782, AAS 103 [2011], 55, 99, 120-121.

64 Cf. AAS 98 [2006], 751, 753, 897, AAS 100 [2008], 76, 93, AAS 101 [2009], 58, AAS 103 [2011], 55.

65 Cf. AAS 99 [2007], 74, 87, 90, AAS 100 [2008], 277, AAS 103 [2011], 652-653.

66 Cf. AAS 97 [2005], 815-816, AAS 98 [2006], 50, 751, 753, AAS 100 [2008], 347, 555, 559, AAS 101 [2009], 696, 870-871, 1040, AAS 102 [2010], 461, AAS 103 [2011], 182, 576, 652-653. 
le altre correnti, paradigmi o rischi per la società, ma chiarisce che cos'è il relativismo ed amplia il discorso. Così il papa indica il suo aspetto ingannevole poiché il «relativismo sembra concederci tutto e in realtà ci svuota» ${ }^{67}$. In un altro brano possiamo notare sia l'approfondimento dei discorsi passati sia la costante preferenza per il singolo e la coscienza. Parlando della verità e libertà il papa definisce un certo atteggiamento come relativismo:

«Avete notato quanto spesso la rivendicazione della libertà viene fatta, senza mai fare riferimento alla verità della persona umana? C’è chi oggi asserisce che il rispetto della libertà del singolo renda ingiusto cercare la verità, compresa la verità su che cosa sia bene. In alcuni ambienti il parlare di verità viene considerato fonte di discussioni o di divisioni e quindi da riservarsi piuttosto alla sfera privata. $\mathrm{E}$ al posto della verità -o meglio, della sua assenza- si è diffusa l'idea che, dando valore indiscriminatamente a tutto, si assicura la libertà e si libera la coscienza. È ciò che chiamiamo relativismo» ${ }^{68}$.

Una diversa formulazione riprende la sostanza dello stesso discorso sul relativismo, il quale non riesce a discriminare fra le opzioni, ma le considera tutte al pari: "Il relativismo, dando valore in pratica indiscriminatamente a tutto, ha reso la "esperienza" importante più di tutto»" ${ }^{69}$. Anche per questo motivo "il relativismo non riesce a vedere l'intero quadro» ${ }^{70}$. Benedetto XVI fa capire anche ai giovani che cos'è il relativismo: "Il relativismo diffuso, secondo il quale tutto si equivale e non esiste alcuna verità, né alcun punto di riferimento assoluto, non genera la vera libertà, ma instabilità, smarrimento, conformismo alle mode del momento» ${ }^{71}$. Questo rilievo, con il quale Benedetto XVI chiarisce, spiega e prolunga la trattazione, farà parte di un elemento conclusivo sul relativismo nel suo pensiero.

6) Quando Benedetto XVI tratta il relativismo legato alla democrazia, allo Stato e alla dignità dell'uomo la trattazione si volge più dettagliata. Brevemente riportiamo le maggiori sottolineature di questo tema. Il relativismo in questa accezione minaccia i lavori della democrazia, nella quale senza un fondamento morale oggettivo non si riesce ga-

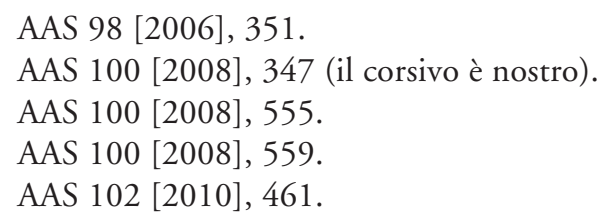


rantire pace e tolleranza ${ }^{72}$. La democrazia, inoltre, considera tutte le posizioni alla pari e per questo rischia di trasformarsi in una dittatura del relativismo, il quale sarebbe il suo elemento costitutivo minacciante la autentica libertà, dignità e la natura stessa dell'uomo ${ }^{73}$. Le discussioni internazionali nel sistema politico contemporaneo sono caratterizzate per la logica relativistica con la quale rifiutano di ammettere la verità sull'uomo e negano un'etica basata sulla legge morale naturale. In questo quadro anche i diritti dell'uomo, che si radicano nella legge naturale e che si caratterizzano per la propria universalità, sono minacciati dalle interpretazioni relativistiche che negano proprio quest'universalità nel nome di condizionamenti sociali, politici e culturali ${ }^{74}$. Se lo Stato non riconosce i principi universali legiferando sulle questioni etiche rischia di cadere nel relativismo, in quanto esso stesso si pone come fonte e principio dell'etica ${ }^{75}$.

7) Altri riferimenti al relativismo. Una volta si afferma che non v'è rischio di cadere nel relativismo per la persona che "è realmente convinta, [che] crede con tutto il cuores ${ }^{76}$. Due volte si fa riferimento all'universalità esclusa ad opera del relativismo ${ }^{77}$. Può darsi che anche il kerygma sia indebolito a causa di questo ${ }^{78}$. Una volta si constata che il relativismo genera la confusione, mentre un'altra volta che esso non aiuta al dialogo interculturale; inoltre, il relativismo è una volta relazionato ai mass-media, mentre il papa ci propone una volta la soluzione del cardinale Newman nei suoi riguardi ${ }^{79}$.

Gli indici negli Insegnamenti di Benedetto XVI annotano nell'arco di cinque anni del suo pontificato dieci riferimenti al termine relativismo, mentre gli indici dell' Enchiridion Vaticanum in tre anni riportano quaranta volte il riferimento allo stesso termine ${ }^{80}$.

72 Cf. AAS 97 [2005], 1046, AAS 103 [2011], 49.

Cf. AAS 97 [2005], 687, AAS 99 [2007], 77, AAS 100 [2008], 315, AAS 101 [2009], 523, AAS 102 [2010], 613.

74 Cf. AAS 99 [2007], 1062, AAS 100 [2008], 334.

75 Cf. AAS 102 [2010], 164.

76 Cf. AAS 97 [2005], 858.

77 Cf. AAS 98 [2006], 705, AAS 100 [2008], 324.

78 Cf. AAS 100 [2008], 342.

79 Cf. AAS 100 [2008], 643, AAS 101 [2009], 661, AAS 102 [2010], 293, 634.

80 Cf. Insegnamenti di Benedetto XVI, I-VI/2, 2005-2010, (Libreria Editrice Vaticana, Città del Vaticano 2006-2011); Enchiridion Vaticanum 23-25, Documenti ufficiali della Santa Sede, 2005-2008, (EDB, Bologna 2008-2011). 


\section{ConClusione}

Formuliamo le conclusioni sul relativismo nel pensiero di Joseph Ratzinger, poi divenuto papa Benedetto XVI, tramite tre elementi elaborati lungo lo sviluppo di questo testo: discorsi e contenuti in cui esso si trova, la frequenza delle occorrenze del termine, il periodo dell'apparizione del termine. Crediamo che grazie a questo testo sono manifeste le ragioni della preferenza di Benedetto XVI per il paradigma e la critica del relativismo. Nel relativismo egli trova un denominatore comune del disagio della società presente. La sua critica del relativismo è orientata sia agli ambiti della società che hanno il potere decisionale -questo è il suo discorso politico-, sia alla cristianità alla quale, nel suo compito di vescovo di Roma, spiega le problematiche riguardanti il relativismo stesso. Nei testi si nota la dialettica con la società secolarizzata, in cui è ad opera la rimozione della dimensione metafisica, etica, spirituale, e infine escatologica. Il relativismo, invece di aprire l'uomo a queste dimensioni che gli appartengono per natura, e nelle quali può trovare il proprio compimento e sviluppo integrale, lascia l'uomo inchinato verso la terra a decidere da solo, o tramite una maggioranza, il proprio destino tragico. Benedetto XVI, nei suoi testi, appare interessato sia a dimostrare l'insufficienza teorica e pratica della posizione relativista, sia ad indicare un percorso da seguire nel cammino della fede cristiana. Paradossalmente, dunque, tutta la pericolosità del relativismo si potrebbe affrontare con una semplice, ma ferma convinzione personale nella scelta fondamentale che coinvolge le altre scelte. Il papa ci indica frequentemente in che cosa dovrebbe consistere questa scelta fondamentale.

Abbiamo mostrato che il relativismo nel pensiero di Joseph Ratzinger appare all'inizio degli anni novanta. Negli stessi anni registriamo l'aumento della sua ricorrenza nel Magistero di Giovanni Paolo II. Il cardinale Joseph Ratzinger sviluppa proprio in quegli anni il discorso sul relativismo in riferimento alla democrazia e ai problemi morali della società democratica. Lo definisce come il paradigma della società contemporanea, dopo che i vecchi paradigmi del marxismo e del liberalismo sono caduti. Sempre negli stessi anni vengono promulgate tre encicliche con il discorso sul relativismo nella società democratica. Successivamente, in un testo della Congregazione per la Dottrina della fede registriamo un ampio discorso con gli stessi contenuti, firmato da Joseph Ratzinger. Però, i suoi articoli sul relativismo e sulla democrazia precedono crono- 
logicamente quelli del Magistero del papa polacco, dove sono trattati gli stessi temi con gli stessi contenuti. I testi del Magistero di Giovanni Paolo II sul relativismo e la democrazia sono inoltre caratterizzati per chiarimenti e spiegazioni, mentre i chiarimenti e le spiegazioni non sono comuni agli altri testi di Giovanni Paolo II sul relativismo, egli solamente constata o riporta il termine. Da tutto ciò concludiamo che tale discorso sul relativismo e sulla democrazia, a cui si associano alcuni altri temi, è proprio di Joseph Ratzinger, anche nei documenti di Giovanni Paolo II.

La preferenza di Benedetto XVI per il concetto del relativismo è confermata dalla frequenza della ricorrenza del termine nel suo pontificato. Essa in pochi anni supera la ricorrenza del termine in ventisei anni del pontificato precedente. In più, abbiamo mostrato che nemmeno vari indici dei testi del Magistero di Giovanni Paolo II recepiscono l'importanza di questo termine nel suo pontificato. Mentre dobbiamo affermare il contrario per il pontificato di Benedetto XVI riguardo agli indici delle stesse collane. Benedetto XVI inoltre tratta il relativismo come qualcosa di unitario, pochissime volte lo specifica con aggettivi come culturale, morale, etico, religioso, intellettuale. Nonostante il termine si trovi nella maggior parte delle occorrenze non specificato, siamo inclini ad affermare che il relativismo che egli tratta è prevalentemente etico; ciò infatti risulta dai problemi etici trattati già negli anni ottanta e il continuo riferimento alla crisi morale della società, che poi associa al relativismo. Tuttavia, nel Magistero del pontificato precedente sono presenti, a partire dagli anni novanta, molti riferimenti al relativismo morale (senza il collegamento con la democrazia). Anche questi si potrebbero attribuire al cardinale Joseph Ratzinger? Soltanto uno studio ancora più approfondito del pensiero di Giovanni Paolo II potrebbe dare una risposta precisa a questa domanda. Ciò però va oltre i limiti e le scelte di questo breve scritto. Ma perché proprio a partire dagli anni novanta e all'avvicinarsi all'anno giubilare abbondano i riferimenti al relativismo nei vari documenti del Magistero? Scopriamo certe risapute caratteristiche di ambedue i personaggi. Joseph Ratzinger è un uomo studioso, riservato e timido, mentre Giovanni Paolo II un avventuriero, un viaggiatore, un attore che pian piano si estingueva già proprio attorno all'anno del gran Giubileo. Sembra che Joseph Ratzinger, con il suo incarico di prefetto della Congregazione per la Dottrina della fede, fose l'uomo più adatto per la redazione dei testi del Magistero; e sembra che il loro Magistero 
198 | Peter Ivanecký

comune anche dopo la morte del papa polacco continui ad orientare gli uomini della società contemporanea, e nei tempi presenti anche sotto le forme di un nuovo Magistero comune con il papa Francesco I. 
Resumen: El papa Benedicto XVI en sus numerosos escritos evocó el concepto del relativismo y la referencia a este término definió su pontificado. En este artículo se quiere mostrar cómo Benedicto desarrolla el tema del relativismo en tres periodos de su actividad intelectual. Al hacer esto se ponen en evidencia sus orígenes: las cenizas del marxismo y las democracias contemporáneas. Uno de nuestros objetivos primarios consiste en demostrar cuál es el tratamiento propio del relativismo de Benedicto en relación a dos tipos de interlocutores: los que toman las decisiones en la esfera pública y los creyentes de la fe cristiana.

Palabras clave: Relativismo, relativismo ético, Benedicto XVI, democracia, marxismo, magisterio.

Abstract: Pope Benedict XVI in his numerous writings evoked the concept of relativism and the reference to this term defined his pontificate. This research will provide valuable information regarding genesis of the Benedict's thought on relativism along three periods of his intellectual activity. This has been done by highlighting its origins: the ashes of Marxism and contemporary democracies. The primary purpose of this study is to manifest Benedict's own treatise of relativism in relation to two types of interlocutors: the decision-makers of the public sphere and Christian believers.

Keyword: Relativism, Ethical Relativism, Benedict XVI, Democracy, Marxism, Magisterium. 
